\title{
Gametogenesis in Liquid Cultures of Chlamydomonas eugametos
}

\author{
By A. M. TOMSON, R. DEMETS, N. P. M. BAKKER, D. STEGWEE \\ AND $H$. VAN DEN ENDE* \\ Plantenfysiologisch Laboratorium, Universiteit van Amsterdam, Kruislaan 318 , \\ 1098 SM Amsterdam, Holland
}

(Receired 3 January 1985; revised 6 March 1985)

\begin{abstract}
At the end of the exponential phase of vegetative growth in liquid cultures, cells of Chlamydomonas eugametos develop into gametes. Gametogenesis can occur without nitrogen deficiency. In continuous light, mating competence lasts only for a few hours, resulting in a low percentage of gametes. Nevertheless, mating competence is considered to be a general property of newly born cells in late exponential phase, since cultivation of both mating-types in mixed cultures normally leads to a high yield of paired cells. No evidence was found for an influence of the presence of partner gametes on gametogenesis. During gametogenesis of the $\mathrm{mt}^{-}$matingtype, extractable biologically active agglutination factor appears in the cell body. Subsequently, after a gametogenic division, mating competence and agglutinability are expressed, both fluctuating synchronously with the light/dark regime.
\end{abstract}

\section{INTRODUCTION}

Chlamydomonas eugametos is a biflagellate unicellular green alga, reproducing vegetatively by sporulation. For sexual reproduction, vegetative cells have to differentiate into gametes. There are no morphological differences between gametes and vegetative cells, nor between the gametes of the two mating-types: plus $\left(m t^{+}\right)$and minus $\left(m t^{-}\right)$. Gametes are distinguished from vegetative cells by their ability to agglutinate and to form vis-à-vis pairs with gametes of the opposite mating-type. Plasmogamy and karyogamy of the paired cells lead eventually to the formation of zygotes. Agglutination takes place via the flagella. The glycoprotein responsible for agglutination (the $m t^{-}$agglutination factor) has been isolated from flagella of $m t^{-}$gametes (Musgrave et al., 1981; Homan et al., 1982), and has also been found in relatively large quantities in the cell body (Pijst et al., 1983).

In the related species $C$. reinhardtii, gametogenesis is induced by nitrogen starvation (Sager \& Granick, 1954). Synchronous gametogenesis is accomplished by placing synchronously dividing vegetative cells in the mid $G_{1}$-phase into nitrogen-free medium (Kates \& Jones, 1964). Gametogenesis usually involves a mitotic division (Kates \& Jones, 1964; Schmeisser et al., 1973). Nitrogen depletion has also been reported to induce sexual differentiation in C.eugametos (Bernstein \& Jahn, 1955), but there are, as far as we know, no reports of synchronous gametogenesis. In nature, at relatively low nitrogen levels, asexual and sexual reproduction of $C$. eugametos seem to co-exist (Trainor, 1975), which prompted us to reinvestigate the role of nitrogen depletion in gametogenesis in this species.

In this work gametogenesis was studied using the following parameters: mating competence, flagellar agglutinability and the presence of extractable biologically active $m t^{-}$agglutination factor.

Abbreviations: LD, $16 \mathrm{~h}$ light $/ 8 \mathrm{~h}$ dark; LL, continuous light; CL-medium, conditioned medium from stationary-phase liquid cultures; CA-medium, conditioned medium from flooded agar cultures. 


\section{METHODS}

Strains. Chlam!domonas eugametos strains UTEX 9 (mating-type plus, $m t^{+}$) and UTEX 10 (mating-type minus, $m t^{-}$) from the Algal (Collection at the University of Texas at Austin, USA, were used.

Plate cultures. (ells were cultivated in Petri dishes on agar as described by Mesland (1976). Standard gamete suspensions were obtained by flooding 2-week-old cultures with water.

Liquid cultures. Cells were cultivated in Fernbach fiasks with $1000 \mathrm{ml}$ medium containing $1 / 100$ of the minerals used by Kates \& Jones (1964), with trace elements according to Wiese (1965) and Fe-EDTA according to Jones (1962). The cultures were grown at $19^{\circ} \mathrm{C}$ with illumination by white fluorescent tubes, $35001 \times\left(42 \mu \mathrm{E} \mathrm{s}^{-1} \mathrm{~m}^{-2}\right)$; a $16 \mathrm{~h} \mathrm{light} / 8 \mathrm{~h}$ dark (LD) regime was used unless indicated otherwise. The cultures were aerated with compressed air and gently agitated at 50 r.p.m. on a rotary shaker. Cells from 2- to 3-week-old agar plates, flooded with medium the previous day, were used as inoculum; the inoculation density was $1 \times 10^{4}$ cells $\mathrm{ml}^{-1}$. To determine cell densities, samples fixed with glutaraldehyde (final concn $1.25 \%, \mathrm{v} / \mathrm{v}$ ) were counted in a haemocytometer.

Mixed cultures. Mixed cultures were started with equal numbers of non-competent $m t^{+}$and $m t^{-}$cells from late stationary phase cultures, to ensure that pairs did not form in the mixed inoculum. Samples, fixed with glutaraldehyde, were counted for single cells and vis-à-vis pairs in a haemocytometer to give both cell density and percentage of paired cells

Mating-type analysis in mixed cultures. To determine the ratio of the two mating-types in a mixed culture, a mouse monoclonal antibody raised against $m t^{-}$flagellar antigens (Homan et al., 1984) was used. This antibody specifically reacted with $m t^{-}$flagella and, after removing the cell wall with $\mathrm{NaOH}$, also with the plasma membrane of $m t^{-}$cells. Therefore, to label all $m t^{-}$cells, including sporulating cells that had retracted their flagella, cells of a mixed culture were fixed with glutaraldehyde and treated with $1 \mathrm{M}-\mathrm{NaOH}$ for $30 \mathrm{~min}$ at $40^{\circ} \mathrm{C}$ according to Pijst $e t$ al. (1983). After washing, the cells were spread on a slide and incubated for $30 \mathrm{~min}$ with the antibody. Bound antibodies were labelled with anti-mouse antibodies bound to fluorescein isothiocyanate (goat anti-mouse IgGFITC, Tago Inc., Burlingame, Calif., USA). The samples were then scored for fluorescent $m t^{-}$cells and nonfluorescent $m t^{+}$cells.

Conditioned medium. Cell suspensions were centrifuged and the supernatants filtered through a $0 \cdot 8 \mu \mathrm{m}$ Millipore filter to obtain conditioned cell-free culture medium.

Mating competence test. To determine the percentage of mating-competent cells in a sample, $m t^{-}$cells were mixed in triplicate in test tubes with an excess of standard $m t^{+}$gametes for $1 \mathrm{~h}$ at $19^{\circ} \mathrm{C}$ with illumination by white fluorescent tubes, $5500 \mathrm{~lx}\left(65 \mu \mathrm{E} \mathrm{s}^{-1} \mathrm{~m}^{-2}\right)$. After fixation with glutaraldehyde, individual cells and vis-à-vis pairs were counted in a haemocytometer. With the known ratio of the two mating types in the mixture, the mating competence of the $m t^{-}$cells (= percentage of $m t^{-}$gametes) was calculated as follows: (no. of pairs $\left.\mathrm{ml}^{-1} \times 100\right) /$ (no. of $m t^{-}$cells $\mathrm{ml}^{-1}$ ). This test, based on an experiment described by Lewin (1956), gives no information about the mating competence of the $m t^{+}$cells forming the majority of the mixture. For correct test conditions, the mating competence of the major partner had to be at least $20 \%$, so the standard $m t^{+}$gametes were always cross-checked as the minor partner against standard $m t^{-}$gametes in a control test. It was established that the optimal test density was $2-4 \times 10^{6}$ cells $\mathrm{ml}^{-1}$, the portion of the minor partner not exceeding $20 \%$ of the mixture.

For determining the mating competence of a $\mathrm{mt}^{+}$suspension, the same procedure was followed with an excess of standard $m t^{-}$gametes.

Test of flagellar agg/utinability. $\mathrm{mt}^{-}$cells were fixed with glutaraldehyde for $30 \mathrm{~min}$, washed, and mixed on a glass slide with standard $m t^{+}$gametes. The agglutinability was compared microscopically with the activity of an equivalent sample of fixed standard $m t^{-}$gametes with a known high mating competence. The agglutinability of the test cells was expressed in relation to the agglutinability of the standard gametes, which was arbitrarily given a value of 5 points.

Extraction and hioassay of $m t^{-}$-agglutination factor. A constant amount of $10^{7} \mathrm{mt}^{-}$cells was exhaustively extracted with $1{ }^{\circ}{ }_{0}(\mathrm{w} / \mathrm{v})$ SDS. The extract was assayed with $m t^{+}$gametes for biological activity by the charcoal technique and the activity was expressed as the titre of the highest dilution of a series $\left(2^{\circ}, 2^{1}, 2^{2}\right.$, etc.) still showing biological activity (Musgrave et al., 1981).

Determination of nitrate and phosphate in the culture medium. The concentration of nitrate in cell-free medium was determined according to Stainton et al. (1974) after quantitative reduction to nitrite in a Cd/Cu-column (Woods $e t$ al., 1967; Nydahl, 1976). Phosphate concentrations were calculated by determining the phosphorus contents of cell-free medium according to Murphy \& Riley (1962).

\section{RESULTS}

\section{Gametogenesis in liquid cultures under continuous light}

The standard method for producing gametes in liquid cultures of $C$. reinhardtii and $C$. moewusii is to transfer vegetative cells from a complete medium to nitrogen-free medium (Kates \& Jones, 1964). When this method was applied to C. eugametos, under the conditions of Kates \& 


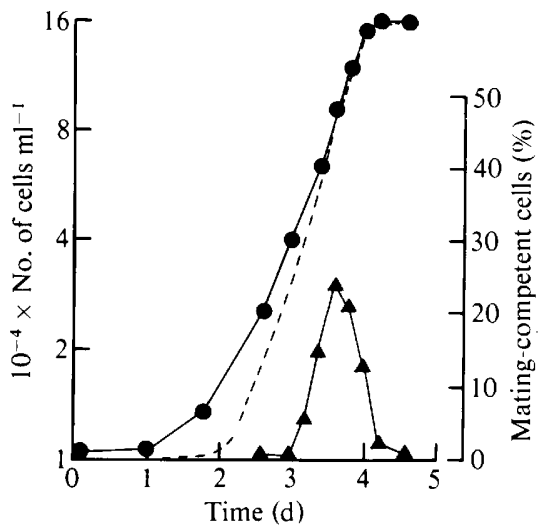

Fig. 1

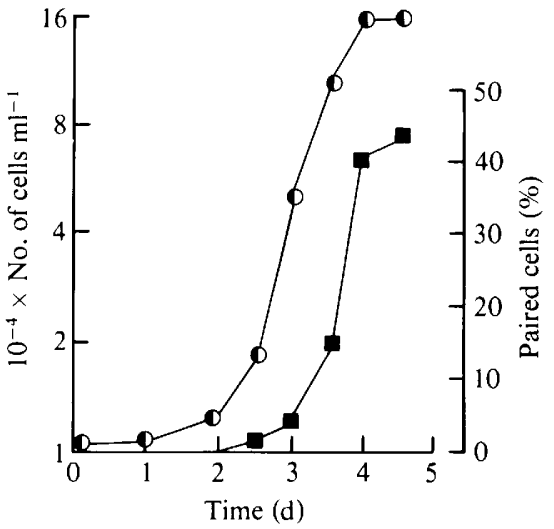

Fig. 2

Fig. 1. Growth ( () and gametogenesis (percentage of mating-competent cells, $\mathbf{\Delta}$ ) of C. eugametos $\mathrm{mt}^{-}$ grown under LL. Results for $m t^{+}$cells were similar, except for the early exponential growth phase (dashed line).

Fig. 2. Growth ( $)$ and gametogenesis (percentage of paired cells,

of C. eugametos grown under LL in a mixed culture $\left(m t^{+}\right.$with $\left.m t^{-}\right)$. Cultures were inoculated with non-competent cells from single cultures in the late stationary phase of growth, to prevent forming of pairs in the inoculum.

Table 1. Growth and nutrient status of C. eugametos $\mathrm{mt}^{-}$with and without additional $\mathrm{KNO}_{3}$

$\begin{array}{lcccc}\text { initial } & \text { final } & \overbrace{\text { initial }}^{\text {innal }} \\ 10^{-4} \times \text { No. of cells } \mathrm{ml}^{-1} & 1 & 28 & 1 & 140 \\ \text { Nitrate concn }(\mu \mathrm{M}) & 11 & 3 \cdot 2 & 1100 & 950 \\ \text { Phosphate concn }(\mu \mathrm{M}) & 4.5 & 0.64 & 4.5 & 0 \cdot 12\end{array}$

Jones (1964), cells kept their vitality and motility, but no gametes were found. Similar results were obtained with cells washed and resuspended in $\mathrm{H}_{2} \mathrm{O}$.

When the mineral content of the medium was decreased (see Methods), gametes were found during 1 or $2 \mathrm{~d}$ at the end of the period of vegetative growth in liquid cultures under continuous light (LL), where they accounted for up to $25 \%$ of the population (Fig. 1). To determine whether the gametogenesis had been due to nitrogen deficiency, the concentration of nitrate in the culture medium was raised from $0.01 \mathrm{~mm}$ to $1 \mathrm{~mm}$ (the concentration used by Kates \& Jones, 1964). Under these conditions, cultures reached a much higher plateau density, indicating that nitrogen depletion caused growth arrest in the normal medium. Mating-competent cells accounted for up to $40 \%$ of the cultures with extra nitrate, which had by no means been exhausted at the end of the growth period (Table 1), indicating that gametogenesis can occur in cultures in which the nitrogen content is not growth limiting. In contrast, only $3 \%$ of the original phosphate was left at the end of growth. Presumably deficiency of some nutrient other than nitrogen had caused the growth arrest and induced gametogenesis.

Although under normal conditions the number of fusing gametes did not exceed $25 \%$, it seemed possible that a higher percentage of the cells did participate in gametogenesis. This was suggested by the appearance and disappearance of the mating-competent cells, which closely corresponded with the production of new cells in the late exponential phase (Fig. 1). The implication is that gametes are mating competent for only a limited period. To test this possibility, $m t^{+}$and $m t^{-}$cells were cultivated in mixed cultures. Assuming that growth and development of the two mating-types occur in parallel and that during the mating-competent period of each particular cell it is possible to find a partner, the prediction is that by 
accumulation the percentage of paired cells should easily exceed $25 \%$. In most experiments, the percentage of paired cells was indeed $40 \%$ or more, with a maximum of $60 \%$ (Fig. 2 ). This result supported the idea of a short mating-competent period per cell, but an alternative explanation for the high gamete yield in mixed cultures could be the mutual stimulation of gametogenesis in both mating-types. However, evidence against this was provided by two deviating cases when hardly any pairs were formed (Fig. 3). Determination of the ratio of the individual mating-types in mixed cultures, using a monoclonal antibody specifically reacting with the $m t^{-}$strain, indicated that in one experiment pair formation failed because $m t^{+}$lagged behind $m t^{-}$in the vegetative growth phase. As a result, there was almost no overlap in the gamete phases, so that few pairs could be formed (Fig. $3 a$ ). Another experiment involved $m t^{-}$cells with a very low mating competence, as determined in the single control culture. In the presence of the opposite mating-type, the $m t^{-}$cells maintained a low mating competence, while the $m t^{+}$strain produced a normal amount of gametes (Fig. $3 b$ ). Therefore, the mating-types seem to develop independently both vegetatively and sexually, without any mutual influence. This leads to the conclusion that mating competence is expressed for only a short period. From the experiment shown in Fig. 1 it was calculated that if all cells born in the late exponential phase underwent gametogenesis, they expressed their mating competence on average for $5 \mathrm{~h}$.

\section{Gametogenesis in liquid cultures under a light/dark regime}

Under LD-conditions sporulation of C. eugametos $m t^{-}$occurred in the dark period, new cells being born in daily bursts at the D-L transition (hatching). Under these conditions gametes were produced during the last cell burst before the culture entered stationary phase (Fig. 4), showing that a gametogenic division was involved. The gamete percentages, though rather variable, were consistently higher than in LL-cultures: up to $80 \%$. Significantly, mating competence was expressed in peaks, in synchrony with the LD-rhythm, with maxima at the D-L transition. Fig. 4 shows three successive peaks of mating competence of 70,60 and $45 \%$, respectively, at constant cell density. This means that the oscillation of the mating competence concerns the same cells, i.e. they lose and recover their mating competence. Cells born during the gametogenic cell division were mating competent for $8 \mathrm{~h}$ on average. This also supports our conclusion that gametes born in continuous light are only temporarily able to fuse. However, since the mating competence returned in the dark period, darkness seems to extend the mating competence. Fig. 4 also demonstrates the relationship between mating competence, agglutinability of the flagella and the presence of extractable, biologically active, agglutination factor. The agglutination factor was first detected in cell extracts towards the end of exponential growth, before the cell burst. After hatching, the daughter cells were immediately agglutinable and mating competent. The flagellar agglutinability fluctuated, like the mating competence, with the LD-rhythm. Both disappeared 2 or $3 \mathrm{~d}$ after the gametogenic division, even though the cells maintained the same amount of extractable agglutination factor. In general, flagellar agglutinability closely followed mating competence, but occasionally strong agglutinability was accompanied by low mating competence, as can be seen on day 6 in Fig. 4 . Therefore a complementary gametic property can be distinguished: fusion competence. Only cells with both flagellar agglutinability and fusion competence are mating competent.

\section{Gametes derived from agar plates}

As inoculum for liquid cultures 2-week-old agar plates were flooded with either $\mathrm{H}_{2} \mathrm{O}$ or complete nutrient medium. Both yielded suspensions with high mating competence. This implies that on 2-week-old agar plates induction to gametogenesis had already occurred. After flooding, the cells generated flagella and became mating competent. When flooded with $\mathrm{H}_{2} \mathrm{O}$, the cells remained mating competent for at least 2 weeks. In complete medium, however, the gametes lost all sexual properties within 1-3 d. When this degametogenesis was complete, vegetative reproduction started (Fig. 4).

Gametes derived from agar plates (standard gametes) contained the same amount of biologically active agglutination factor per cell as gametes in liquid cultures (Fig. 4). However, the prolonged mating competence of standard gametes strongly contrasted with that of the 

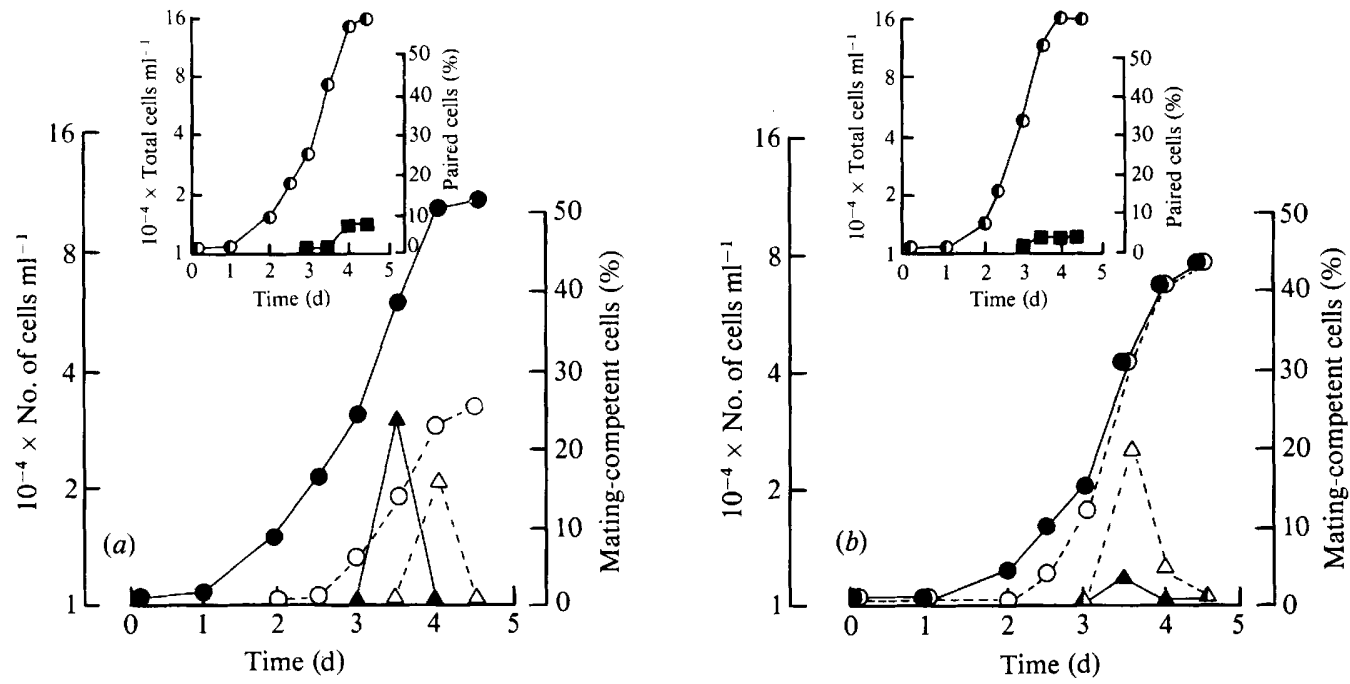

Fig. 3. Growth and gametogenesis of $C$. eugametos during two experiments ( $a$ and $b$ ) similar to that of Fig. 2, but where forming of pairs was negligible (insets). In experiment (a) the development of $m t^{+}$ lagged behind that of $m t^{-}$, so that few pairs could be formed. In experiment (b) $m t^{-}$had a low mating competence compared to its partner, leaving most $m t^{+}$gametes unmated. The separate growth patterns of the $m t^{+}$and $m t^{-}$cells in one culture were measured by determining the ratio of the mating-types using a $m t^{-}$-specific monoclonal antibody (see Methods). The percentage of competent $m t^{+}$cells was determined by adding an excess of standard $m t^{-}$gametes to culture samples and scoring the number of pairs formed. The percentage of competent $m t^{-}$cells was determined in a similar way by adding standard $m t^{+}$gametes. $\mathrm{O}$, Growth of $m t^{+} ; \mathbf{O}$, growth of $m t^{-} ; \mathbf{D}$, growth of whole culture; $\mathbf{\square}$ percentage of paired cells; $\triangle$, percentage of mating-competent $m t^{+}$cells; $\boldsymbol{\Delta}$, percentage of matingcompetent $m t^{-}$cells.

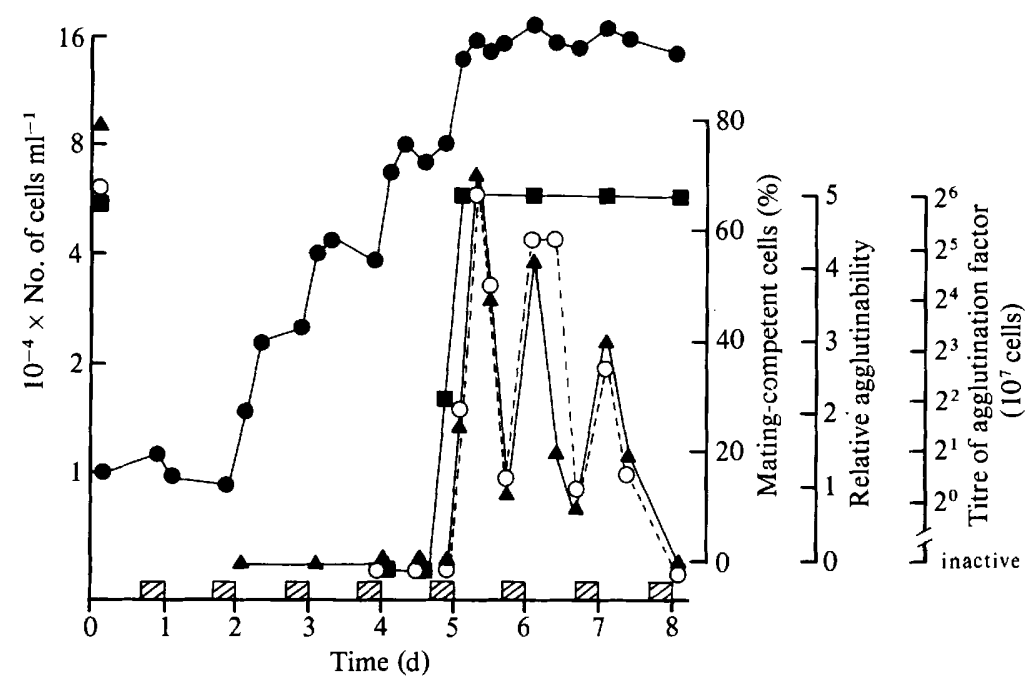

Fig. 4. Growth and gametogenesis of $C$. eugametos $m t^{-}$grown under a circadian LD-rhythm. Dark periods are represented as hatched rectangles. Cultures were inoculated with cells derived from agar plates. $\boldsymbol{O}$, Growth; $\boldsymbol{\Delta}$, percentage of mating-competent cells; $\bigcirc$, relative agglutinability; $\boldsymbol{\square}$, titre of $m t^{-}$agglutination factor in bioassay.

liquid-induced gametes. To investigate whether this difference was caused by the medium, standard $m t^{-}$gametes were washed and resuspended in conditioned medium from liquid cultures in the stationary phase of growth (CL-medium) and from agar cultures flooded with water (CA-medium) and kept on a LD-regime. The mating competence was determined in both 


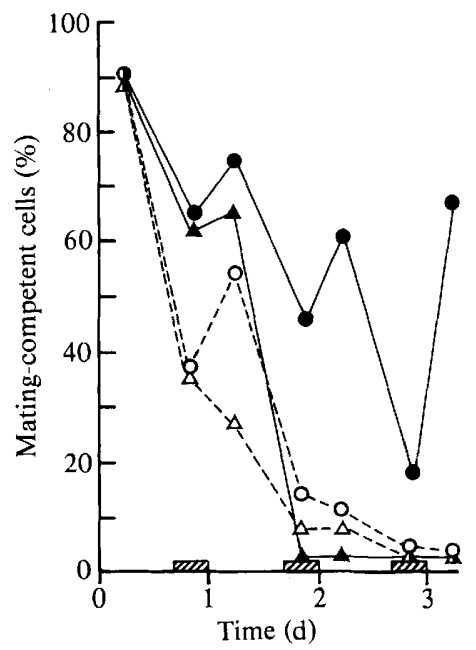

Fig. 5. Mating competence of standard $m t^{-}$gametes under a LD-regime, suspended in conditioned medium from liquid cultures (CL; open symbols) and from agar cultures flooded with water (CA; filled symbols). Dark periods are represented as hatched rectangles. Gametes were suspended at densities of $1.5 \times 10^{7} \mathrm{ml}^{-1}(\mathrm{O}, 0)$ and of $2.5 \times 10^{5} \mathrm{ml}^{-1}(\Delta ; \triangle)$. Cell densities were constant throughout the experiment.

the dark and the light periods. When gametes were resuspended at high density (about $1.5 \times 10^{7}$ cells $\mathrm{ml}^{-1}$, i.e. the normal cell density of agar-derived gamete suspensions), the mating competence in CA-medium was preserved throughout the experiment and showed a LD-rhythm similar to that of liquid-induced gametes (Fig. 5). In CL-medium, however, mating competence soon dropped and was lost within $3 \mathrm{~d}$. When gametes were resuspended at low density (about 2.5 $\times 10^{5}$ cells $\mathrm{ml}^{-1}$, i.e. the normal cell density of liquid-induced gamete suspensions), mating competence in CA-medium dropped rapidly and disappeared within $2 \mathrm{~d}$. In CL-medium gametes lost their mating competence immediately and behaved like gametes induced in this medium. In all cases cell densities were constant throughout the experiment. These results indicate that conditioned medium from agar cultures contains substances that maintain the mating competence, but only when the cell density is high. In our liquid cultures the cell density is too low to produce enough of these substances to preserve the mating competence for a long time.

\section{DISCUSSION}

Gametogenesis in C.eugametos seems not to be triggered by the same events as gametogenesis in $C$. reinhardtii and $C$. moewusii. Cells could not be induced to undergo gametogenesis by transferring them to nitrogen-free medium. Furthermore, gametogenesis not only occurred when nitrate was at growth-limiting concentration, but also when nitrate was abundant and growth was prevented by deficiency of some other nutrient. These findings indicate that, as suggested by Trainor (1975), nitrogen does not play a key role in the gametogenesis of $C$. eugametos. Our conclusion is that nutrient stress in general is the factor that induces cells to undergo a final and gametogenic division. Wiese (1984) has also argued that gametogenesis is not induced by nitrogen depletion and that nitrate does not prevent gametogenesis, claiming that ammonium depletion rather than nitrate depletion triggers gametogenesis in C. eugametos. However, it is obvious that this was not the case in our experiments.

In the colonial Volvocales Gonium and Pandorina, gametogenesis only occurs after mixing the two mating types (Wiese, 1969). Our study did not provide any evidence for a mutual influence on vegetative or sexual development of the two mating-types.

In LD-cultures gametes appeared during the last cell burst. So, as in C. reinhardtii, gametogenesis in C. eugametos involves a gametogenic division (Kates \& Jones, 1964; 
Schmeisser et al., 1973). Agglutination factor could be detected in the daughter cells prior to hatching. Gametes in liquid cultures contained the same amount of agglutination factor as gametes derived from agar plates. This may mean that gametes contain a specific amount of agglutination factor per cell. Only a small part of the agglutination factor is extracted from the flagella, most of it being located on the cell body, as shown by Pijst et al. (1983). These authors proposed that agglutination factor could perhaps diffuse from the cell surface to the flagella and that the cell body therefore acted as a pool. However, the fact that many of our cells contained a full complement of agglutination factor, but were not agglutinable, suggests that there is no free diffusion between the membranes of the cell body and the flagella.

Flagellar agglutinability and mating competence of gametes oscillated with the LD-rhythm. Since the decline in flagellar agglutinability always occurred after the onset of the light period, a possible explanation is that light has a negative effect on this property and consequently on mating competence. This effect was only partly reversible and after 2 or $3 \mathrm{~d}$ no matingcompetent cells were left. The short period of mating competence found in cultures grown under continuous light can also be seen as a result of the negative effect of light. This loss of mating competence must be distinguished from degametogenesis resulting in vegetative growth. Gametes induced in liquid cultures, though losing their mating competence, did not revert to the vegetative state. This is concluded from the absence of cell division and the presence of agglutination factor.

Under a LD-regime, liquid-induced gametes rhythmically expressed their mating competence for only a few days. In contrast, agar-derived gametes in medium from flooded agar cultures preserved this rhythmic mating competence, but only at a high cell density, indicating that substances secreted by the cells play some role. Taking this phenomenon together with the oscillation of flagellar agglutinability, an alternative explanation for the fluctuation in mating competence is that it is caused by a rhythm in the secretion of such substances, taking place only in the dark period, followed by a breakdown, not necessarily restricted to the light period as suggested above. An interesting question is to what extent transport of agglutination factor from the cellular pool via the flagella into the medium takes place. Agar-derived $m t^{-}$gametes excrete large amounts of agglutinative membrane vesicles, so-called isoagglutinins (Homan, 1981), and these may be needed for the re-establishment of the flagellar agglutinability.

The authors would like to thank Mrs C. A. M. Sigon for her assistance in the course of this investigation, MrK. M. Royackers of the Laboratory of Aquatic Ecology, University of Amsterdam, for determining nitrate and phosphate contents, Dr A. Musgrave for helpful suggestions and Dr W. L. Homan for supplying monoclonal antibodies.

\section{REFERENCES}

Bernstein, E. \& JAHN, T. L. (1955). Certain aspects of sexuality of two species of Chlamydomonas. Journal of Protozoology 2, 81-85.

Homan, W. L. (1981). Sexual cell recognition in Chlamydomonas eugametos. In Lectins - Biology, Biochemistry, Clinical Biochemistry, vol. I, pp. 51-58. Edited by T. C. Bøg Hansen. Berlin \& New York: Walter de Bruyn.

Homan, W. L., Gijsberti Hodenpijl, P., Musgrave, A. \& ENDE, H. VAN DEN (1982). Reconstitution of biological activity in isoagglutinins from Chlamydomonas eugametos. Planta 155, 529-535.

Homan, W. L., Kalshoven, H., Nederbragt, A., KolK, A. H. J. \& ENDE, H. VAN DEN (1984). Monoclonal antibodies against flagellar glycoproteins of Chlamydomonas eugametos. 6th John Innes Symposium, Norwich, UK, abstract 14.

JONES, R. F. (1962). Extracellular mucilage of the red alga Porphyridium cruentum. Journal of Comparative and Cellular Physiology 61, 61-64.
Kates, J. R. \& Jones, R. F. (1964). The control of gametic differentiation in liquid cultures of Chlamydomonas. Journal of Comparative and Cellular Physio$\log y$ 63, $157-163$.

LEWIN, R. A. (1956). Control of sexual activity in Chlamydomonas by light. Journal of General Microbiology 15, 170-185.

Mesland, D. A. M. (1976). Mating in Chlamydomonas eugametos. A scanning electron microscopical study. Archives of Microbiology 109, 31-35.

MurPhy, J. \& Riley, J. P. (1962). A modified single solution method for the determination of phosphate in natural waters. Analytica chimica acta 26, 31-36.

Musgrave, A., Eijk, E. van, Te Welscher, R., Broekman, R., Lens, P. F., Homan, W. L. \& Ende, H. VAN DEN (1981). Sexual agglutination factor from Chlamydomonas eugametos. Planta 153 362-369.

NYDAHL, F. (1976). On the optimum conditions for the reduction of nitrate to nitrite by cadmium. Talanta 23, 349-357. 
Piust, H. L. A., Zilver, R. J., Musgrave, A. \& Ende, H. VAN DEN (1983). Agglutination factor in the cell body of Chlamydomonas eugametos. Planta 158, 403409.

SAGER, R. \& GRANICK, S. (1954). Nutritional control of sexuality in Chlamydomonas reinhardi. Journal of General Physiology 37, 729-742.

Schmeisser, E. T., Baumgartel, D. M. \& Howell, S. H. (1973). Gametic differentiation in Chlamydomonas reinhardi: cell cycle dependency and rates in attainment of mating competence. Developmental Biology 31, 31-37.

Stainton, M. P., Capel, M. J. \& Armstrong, F. A. J. (1974). The chemical analysis of freshwater. Miscellaneous Special Publication. Research and Development Directorate Freshwater Institute, Winnipeg, Manitoba, no. 25.

Trainor, F. R. (1975). Is a reduced level of nitrogen essential for Chlamydomonas eugametos mating in nature? Phycologia 14, 167-170.
WIESE, L. (1965). On sexual agglutination and mating type substances (gamones) in isogamous heterothallic chlamydomonads. I. Evidence of the identity of the gamones with the surface components responsible for sexual flagellar contact. Journal of Phycology 1, 46-54.

WIESE, L. (1969). Algae. In Fertilization $I I$, pp. 135188. Edited by C. B. Metz \& A. Monroy. London \& New York: Academic Press.

WIESE, L. (1984). Mating systems in unicellular algae. In Cellular Interactions, pp. 238-260. Edited by H. F. Linskens \& J. Heslop-Harrison. Berlin: SpringerVerlag.

Woods, E. D., Armstrong, F. A. J. \& Richards, F. A. (1967). Determination of nitrate in sea water by cadmium copper reduction to nitrite. Journal of the Marine Biological Association of the United Kingdom 47, 23-31. 